\title{
Sécheresse hydrologique : quelles conséquences ?
}

\author{
Thierry POINTET
}

SHF

L'hiver 2016-2017 a été marqué par un déficit pluviométrique exceptionnel sur le second semestre 2016 et des pluies variables d'une région à l'autre en 2017. C'est l'une des 4 années hydrologiques les moins arrosées depuis 1959, année du début des relevés réguliers des précipitations sur un grand nombre de stations. ${ }^{*}$

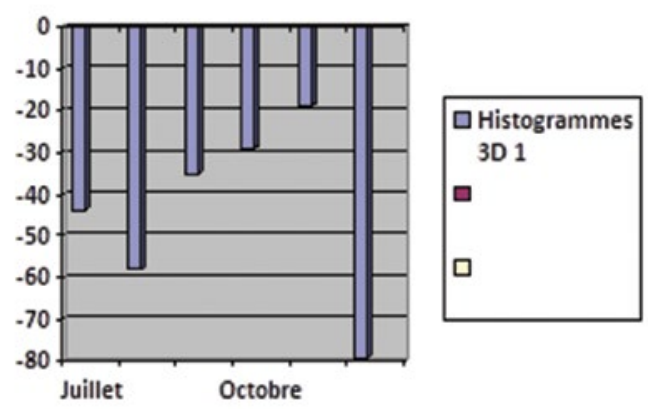

Déficit des pluies mensuelles (juillet à décembre) sur l'ensemble de la France exprimé en \% par rapport à la normale.

Conséquence de cette faible pluviométrie, le déficit enregistré sur les pluies efficaces entre septembre 2016 et février 2017 est compris entre 25 et $75 \%$ sur l'ouest et le nord-est du pays. Il est encore supérieur à $25 \%$ sur le nord-est, le Jura et le nord des Alpes. En avril, il s'est atténué au sud de la Seine.**
홉

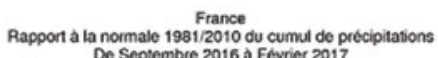

De Septembre 2016 a Fevrier 2017

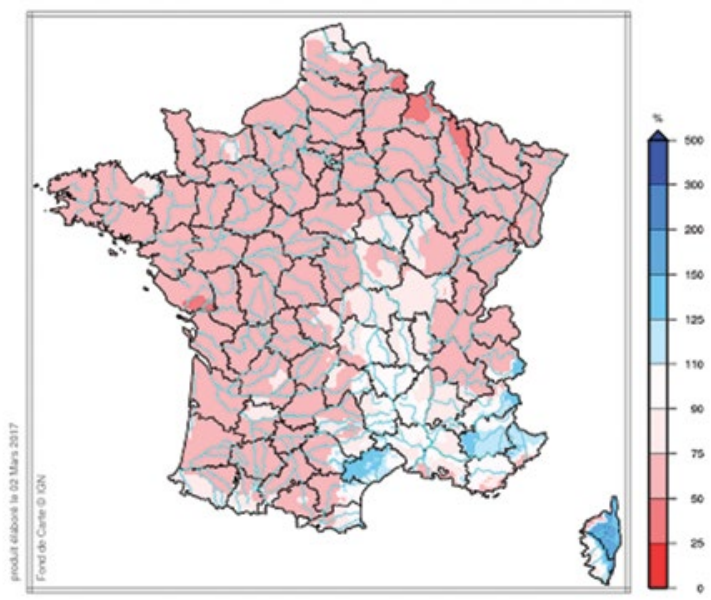

Source Eaufrance

Avec un degré d'inertie supplémentaire, la faible recharge hivernale des nappes conduit à des niveaux au mieux proches de la normale sur la plus grande partie du territoire, et des niveaux très bas pour les grandes nappes du sud-ouest. Autre trait inhabituel, en dynamique cette fois, bon nombre de nappes à forte et même à faible inertie n'ont pas encore bénéficié de la recharge hivernale que l'on observe habituellement dès le printemps (figurés carrés). Seule une moitié des

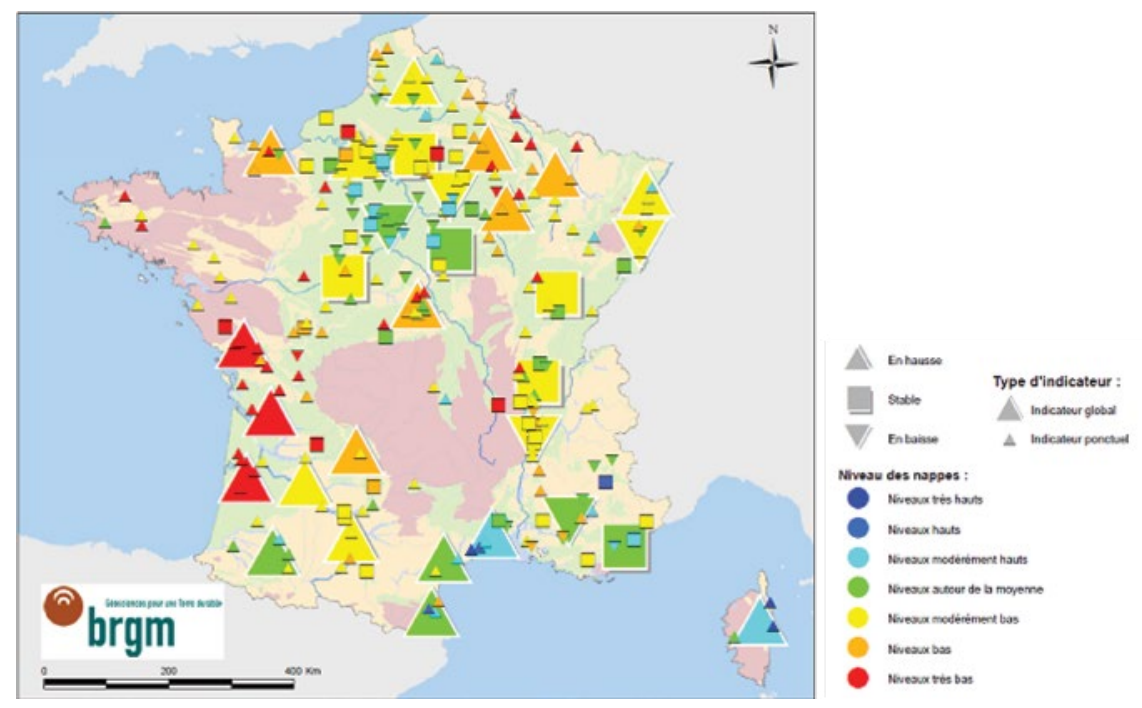

Etat des nappes au 1-3-2017 qui montre une situation au mieux normale sur la plus grande partie de la France continentale. Une partie du sud du Bassin parisien et le pourtour méditerranéen présentent des niveaux de nappes voisins de la moyenne voire modérément hauts. A défaut de grandes nappes sur le massif armoricain, les petites nappes y sont très basses. ***

*. Source Météo France

***. Source BRGM

**. Source Eaufrance (cf. Agence française pour le biodiversité) 
points d'observation attestent d'une remontée des niveaux (les triangles à sommet dirigé vers le haut).

Les ouvrages destinés au soutien d'étiage des cours d'eau sont en revanche remplis à $80 \%$.

Dans les régions en déficit, particulièrement en l'absence de nappes profondes, il faut s'attendre l'été prochain à des répercussions sur les ressources habituellement sollicitées, avec les conséquences logiques sur les usages. Le soutien des nappes au débit des rivières, hors période de pluies, devrait être inférieur à la normale, les cours d'eau temporaires tariront plus tôt, le partage des ressources dans les zones de répartition sera précoce, le rôle des grandes rivières pour assimiler les eaux de refroidissement des centrales au fil de l'eau sera délicat, etc.

On ne peut encore formuler de prévision pour l'agriculture, qui utilise d'abord l'eau des sols, réservoir à faible inertie dont le fonctionnement est lié à la pluviométrie de l'instant.

\section{V'GICRUES}

Le service central d'hydrométéorologie et d'appui à la prévision des inondations (Schapi) est un service à compétence nationale du ministère de la transistion écologique et solidaire, rattaché à la direction générale de la prévention des risques et localisé à Toulouse.

Le Schapi produit et diffuse, 2 fois par jour et davantage si nécessaire, une information de vigilance sur les crues (cartes, prévisions et bulletins www.vigicrues.gouv.fr), en collaboration avec les 19 services de prévision des crues (SPC) métropolitains. II transmet également cette information à Météo-France pour assurer la coproduction des volets " pluie- inondation » et « inondation » de la vigilance météorologique.

Le Schapi assure également la mise à disposition, pour les professionnels et le grand public, de toutes les données disponibles dans la base nationale des données hydrométriques (Banque HYDRO : hauteurs d'eau et débits sur plus de 3000 points de mesure dans les principaux cours d'eau - www.hydro.eaufrance.fr).

Le Schapi pilote le réseau VIGICRUES, qui regroupe les SPC, les 25 unités d'hydrométrie et les cellules de veille hydrologique dans les DOM et en Corse, en définissant et en contribuant à mettre en œuve la politique nationale dans ces domaines. II œurre à l'harmonisation des matériels, des pratiques opérationnelles et des méthodes, pour la prévision des crues et des inondations comme pour l'hydrométrie, et pilote le développement, qu'il assure en partie, d'outils d'intérêt commun pour le réseau. Enfin, le Schapi a également un rôle prospectif : il prépare notamment des réponses réalistes aux évolutions fortes de la demande sociale en matière. II pilote également la mission de référent départemental pour l'appui technique à la préparation et à la gestion de crise d'inondation (Mission RDI)

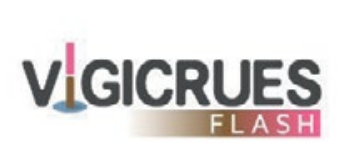

En mars 2017, un nouveau service complémentaire à Vigicrues a été ouvert. VIGICRUES Flash, service automatique d'avertissement sur les crues soudaines couvre le réseau non surveillé sur $30000 \mathrm{~km}$ de cours d'eau et concerne 10000 communes. Il complete le dispositive

existant des APIC (Avertissements Pluies Intenses à l'échelle des Communes) mis en place par Météo-France et partage d'ailleurs le même portail d'accès, offrant ainsi aux communes et aux services de l'État un outil plus complet pour faire face aux phénomènes de crues rapides.

Plus d'infos : https://apic.meteo.fr/ressources/doc/doc mairie.pdf www.developpement-durable.gouv.fr 